\section{Usos del coeficiente de alfa de Cronbach}

En un artículo recientemente publicado en Biomédica se informa sobre la calidad de vida y las condiciones de salud de la población adulta mayor en Medellín. Es un aporte importante al conocimiento de las condiciones de vida de un grupo poblacional creciente. No obstante, no hay suficiente claridad sobre la forma en que se midió la calidad de vida. Se informa que la independencia, la economía y la seguridad social y las redes de apoyo social explican un $97,4 \%$ de la variabilidad total según el alfa de Cronbach. Tampoco queda claro cómo se hizo el análisis factorial (1).

Los resultados sobre la calidad de vida sugieren que se usó una escala para cuantificarla y se informó el coeficiente de alfa de Cronbach; no obstante, se dio un porcentaje (1). Se recomienda informar el valor del alfa de Cronbach siempre que se use una escala, aunque haya sido formalmente validada (2). El coeficiente de alfa de Cronbach se usa para conocer la consistencia interna de una escala, es decir la correlación entre los ítems, y para establecer la homogeneidad (3). Este coeficiente oscila entre $-1,0$ y 1,0 y se considera que la consistencia interna es alta si se encuentra entre 0,70 y 0,90 . Los valores inferiores a 0.70 indican una baja consistencia interna y los superiores a 0.90 sugieren que la escala tiene varios ítems que miden exactamente lo mismo 0 que está compuesta por más de veinte ítems (4).

Asimismo, se observa que la suma de varianza explicada por los factores presentados es mayor a 100\%. En el análisis factorial de una escala, el o los factores importantes identificados excepcionalmente explican más del $60 \%$ de la varianza, dado que este porcentaje es igual al autovalor dividido por el número de ítems que forman la escala (5).

Es necesario precisar la forma en que se valoró la calidad de vida de los adultos mayores y si se usó una escala, informar correctamente el comportamiento psicométrico de la misma.
Adalberto Campo-Arias, MD, Instituto de Investigación del Comportamiento Humano ww w. comportamientohumano.org Transversal 93 No. 53-48 Interior 68 Fax 57122319 57, Bogotá, D.C.

\section{Referencias}

1. Cardona D, Estrada A, Agudelo HB. Calidad de vida y condiciones de salud de la población adulta mayor de Medellín. Biomédica 2006;26:206-15.

2. Cortina JM. What is coefficient alpha? An examination of theory and applications. J Applied Psychol 1993;78:98-104.

3. Cronbach LJ. Coefficient alpha and the internal structure of test. Psychometrika 1951;16:297-334.

4. Oviedo HC, Campo-Arias A. Aproximación al uso del coeficiente alfa de Cronbach. Rev Colomb Psiquiatr 2005;34:572-80.

5. Streiner DL. Figuring out factors: the use and misuse of factor analysis. Can J Psychiatry 1994;39:135-40.

\section{Respuesta}

Por medio de esta carta se da respuesta a las inquietudes manifestadas por el doctor Adalberto Campo-Arias en la comunicación enviada al editor de la revista Biomédica sobre la valoración de la calidad de vida de la población adulta mayor y el uso del coeficiente de alfa de Cronbach. Para tal fin, se procederá en el orden en que emergieron las inquietudes del doctor Campo-Arias.

1. En el primer párrafo se hace referencia a "...no hay claridad sobre la forma en que se midió la calidad de vida (...). De la misma forma, no es claro como se hizo el análisis factorial").

En lo relacionado a la forma de medir la calidad de vida, los investigadores tuvimos en cuenta lo multifactorial del concepto, que generalmente se compone de elementos netamente económicos, pero como se ha demostrado*, en su valoración

\footnotetext{
* La Organización Mundial de la Salud (OMS) definió la calidad de vida como: "la percepción individual de la propia posición en la vida dentro del contexto del sistema cultural y de valores en que se vive y en relación con sus objetivos, esperanzas, normas y preocupaciones".
} 
se incluyen otros aspectos importantes y necesarios (1), por lo que se optó por su análisis a través de componentes $\left({ }^{2}\right)$ como el económico, el social, el de salud y seguridad social (de los cuales se enuncian algunas de las variables consideradas), análisis éste que fue univariado, bivariado y multivariado dentro y entre cada componente, sin utilizar ninguna escala en su medición; por ello en ningún momento se mencionó ni se calculó algún índice de calidad de vida, puesto que se buscaba describir las características que permitían esbozar, de alguna manera, cómo estaba la calidad de vida de los adultos mayores a través de algunas características de cada componente analizado.

Por otro lado, en lo relacionado al análisis factorial, en materiales y métodos se enuncia que el procedimiento utilizado para el modelo fue el Prinqual con el fin de poder hacer el análisis multivariado a través de componentes principales. El procedimiento de cuantificación óptima (Optimal Scaling) o escalamiento óptimo ** se utiliza cuando las variables que ingresan son de naturaleza categórica, entonces se deben hacer adaptaciones y así proceder a aplicar un modelo factorial; el uso de datos categóricos es frecuente en estudios de mercado, encuestas poblacionales e investigación en ciencias sociales y del comportamiento. De hecho, los modelos estándar que permiten analizar específicamente datos categóricos, con frecuencia no funcionan bien en conjuntos de datos con las siguientes características: observaciones insuficientes, demasiadas variables y demasiados valores por cada variable.

La idea de esta adecuación es que las variables que se van a cuantificar puedan tener relaciones no lineales independientemente de cómo se midan. Las técnicas más utilizadas son el análisis de correspondencias, el análisis de correspondencias múltiple, el análisis de componentes principales categórico y el análisis de correlación canónica

** Escalamiento óptimo es asignar cuantificaciones numéricas a las categorías de cada variable, lo que permite utilizar los procedimientos estándar para obtener una solución con las variables cuantificadas. Los valores de escala óptimos se asignan a las categorías de cada variable de acuerdo con el criterio de optimización del procedimiento que se esté utilizando. no lineal, que corresponden al análisis de datos multivariantes conocido como reducción de dimensiones. Para este estudio se escogió el análisis de componentes principales categórico, que es el adecuado cuando se desea tener en cuenta los patrones de variación de un único conjunto de variables con varios tipos de niveles de escalamiento óptimos. Esta técnica intenta reducir la dimensionalidad de un conjunto de variables, al mismo tiempo que tiene en cuenta toda la variación que sea posible. Se asignan valores de escala a cada categoría de cada variable, de manera que estos valores sean óptimos respecto a la solución de componentes principales. Los objetos del análisis reciben puntuaciones de componentes en función de los datos cuantificados.

La solución de un análisis de componentes principales categórico maximiza las correlaciones de las puntuaciones de objetos con cada una de las variables cuantificadas para el número de componentes (dimensiones) especificado. Ahora bien, si todas las variables se escalan a nivel numérico, el análisis de componentes principales categórico es equivalente al análisis de componentes principales estándar; es decir, el análisis de componentes principales categórico es una alternativa al cálculo de las correlaciones entre las escalas no numéricas y su análisis mediante un enfoque de análisis de factores o de componentes principales estándar.

El objetivo de los análisis de componentes principales es la reducción de un conjunto original de variables en un conjunto más pequeño de componentes no correlacionados que representen la mayor parte de la información encontrada en las variables originales y es la técnica más útil cuando un extenso número de variables impide una interpretación eficaz de las relaciones. Al reducir la dimensionalidad, se interpreta un pequeño número de componentes en lugar de un extenso número de variables. El análisis típico de componentes principales asume relaciones lineales entre las variables numéricas, mientras el método de cuantificación óptima permite escalar las variables a diferentes niveles, permitiendo modelar relaciones no lineales entre ellas (3). 
2. Respecto a las inquietudes en el segundo párrafo, el señor Campo-Arias dice, "Los resultados sobre la calidad de vida sugieren que se usó una escala para cuantificarla y se informó el coeficiente de alfa de Cronbach; no obstante, se dio un porcentaje".

Ya se explicó lo relacionado con la medición utilizando el procedimiento de cuantificación óptima, pues permite escalar las variables a diferentes niveles; es decir, no se utilizó ninguna escala en la valoración de la calidad de vida de la población adulta mayor, tampoco se validó ninguna escala ni, como lo sugiere el doctor Campo, se recomendó informar el valor del alfa de Cronbach siempre que se use una escala para determinar su consistencia interna, situación que no es el caso en este articulo. En nuestra investigación se usó el alfa de Cronbach resultante de la discretización de los datos en la adecuación del modelo de componentes principales para el análisis factorial.

El análisis factorial consta de cuatro fases características: cálculo de una matriz capaz de expresar la variabilidad conjunta de todas las variables, la extracción del número óptimo de factores, la rotación de la solución para facilitar su interpretación y la estimación de las puntuaciones. En la segunda fase, la extracción de factores se puede hacer a través de diferentes métodos como los de componentes principales, mínimos cuadrados no ponderados, mínimos cuadrados generalizados, máxima verosimilitud, ejes principales, alfa o imagen; para este estudio se utilizó el método de componentes principales, en el que los factores obtenidos son los autovalores de la matriz de correlaciones re-escalados, y el de alfa de Cronbach, que considera las variables incluidas en el análisis como una muestra del universo de las variables posibles, es decir, maximiza la posibilidad de generalizar los factores (4).

De otro lado, no compartimos la afirmación en torno a que el alfa de Cronbach es un valor que oscila entre -1 y 1 , pues como lo enuncian algunos autores, es un valor que oscila entre 0 y $1(5,6)$. Sí estamos de acuerdo cuando informa que valores por debajo de 0,7 indican baja consistencia interna y que valores por encima de 0,9 pueden indicar que algunos ítems están midiendo lo mismo; pero no es comprensible afirmar que valores por encima de 0,9 indican que la escala tiene más de 20 ítems, pues equivaldría a decir que muchas validaciones realizadas, principalmente en salud, están fallando, pues sus alfas son mayores a 0,9 , o que no se pueden evaluar escalas de más de 20 ítems.

Esta situación se ve refutada al leer algunos artículos científicos como el estudio "Validación de un instrumento para medir calidad de vida en hemodiálisis crónica: perfil de impacto de la enfermedad", el cual evaluó un test de 136 ítems cuyos alfas son valores inferiores a 0,9 (7). Igual situación se encontró en el informe de González RA (8). A modo de ilustración se citan otros artículos, los cuales obtuvieron alfas por encima de 0,9 (9-12) y manejan más de 20 ítems (Todas las validaciones del SF-36 y del body shape Questionnaire (34 ítems), tan utilizados en calidad de vida y problemas de la conducta alimentaria respectivamente) $(13,14)$.

Reconocemos que el alfa citado en el artículo debió colocarse según el valor del coeficiente $(0,974)$ e igual con los otros tres valores, pues así los reporta la mayoría de la literatura. Pero consideramos que perfectamente se puede expresar en porcentaje, como sucede con la sensibilidad, la especificad y el coeficiente de determinación, que son valores que comúnmente se citan en los artículos en forma de proporción.

3. En el penúltimo párrafo, el doctor Campo-Arias dice, "... la suma de la varianza explicada por los factores presentados es mayor a 100\%”, y tiene razón, pero en nuestro artículo, los tres valores mencionados en el apartado sobre análisis de componentes principales (calidad de vida: 91,7 , $83,9,79,4 \%$ ) corresponden al peso de estas variables dentro de cada uno de componentes, independientes unos de otros, pues el coeficiente que los agrupa a todos fue de 0,974.

Es decir, estos valores no se suman para generar el coeficiente total sino que son los valores alfa para cada uno de los componentes y así lo redactamos en el artículo: "Los componentes que mejor explican la calidad de vida de los adultos mayores de la ciudad de Medellín son la 
independencia, la economía y la seguridad social y las redes de apoyo social; estos tres explican en un $97,4 \%$ (según el alfa de Cronbach) la variabilidad total de la calidad de vida de los adultos mayores. De esta forma se encontró que las variables relacionadas con la independencia explican en un $91,7 \%$ el comportamiento de este componente; las variables económicas y de seguridad social explican en un $83,9 \%$ dicho componente, y las variables de las redes de apoyo social lo explican en un $79,4 \%$. Las variables objetivas referentes a las condiciones materiales de la vivienda del adulto mayor fueron excluidas por el modelo."

De esta forma, esperamos haber dado respuesta satisfactoria a las inquietudes del lector sobre la forma en que se valoró la calidad de vida de los adultos mayores en esta investigación, el modelo multivariado de análisis factorial con el método de componentes principales de datos categóricos y la cuantificación o escalamiento óptimo empleado, la no aplicación de ninguna escala en su medición $y$, por ende, la imposibilidad de presentar su comportamiento psicométrico.

Atentamente,

Doris Cardona Arango

Alejandro Estrada Restrepo

Héctor Byron Agudelo García

Autores

\section{Referencias}

1. Organización Mundial de la Salud. Programa Envejecimiento y Ciclo Vital. Envejecimiento activo: un marco político. Rev Esp Geriatr Gerontol 2002;37:74105.

2. Fleury E, Lana da Costa C. Qualidade de vida e saúde: aspectos conceituais e metodológicos. Cad Saúde Pública 2004;20:580-8.
3. Meulman JJ, Heiser WJ. Programa SPSS Categories. Chicago: SPSS Inc.; 1990.

4. SPSS Inc. Programa SPSS. Análisis factorial. Categories. Chicago: SPSS Inc.; 1990.

5. Polit DF, Hungler BP. Investigación científica en ciencias de la salud. México: McGraw Hill Interamericana; 2000.p. 397.

6. Bland JM, Altman DG. Cronbach's alpha. Br Med J 1997;314:572.

7. Schwartzmann L, Olaizola I, Guerra A, Dergazarian S, Francolino C, Porley G, et al. Validación de un instrumento para medir calidad de vida en hemodiálisis crónica: Perfil de impacto de la enfermedad. Rev Med Uruguay 1999;15:103-9.

8. Alonso R, Bayarre H, Artiles L. Construcción de un instrumento para medir la satisfacción personal en mujeres de mediana edad. Rev Cubana Salud Pública 2004;30.

9. Suh GH, Kang CJ. Validation of the severe impairment battery for patients with Alzheimer's disease in Korea. Int J Geriatr Psychiatry 2006;21:626-32.

10. Pachana NA, Byrne GJ, Siddle H, Koloski N, Harley E, Arnold E. Development and validation of the geriatric anxiety inventory. Int Psychogeriatr 2007;19:10314.

11. Ertan FS, Ertan T, Kiziltan G, Uygucgil H. Reliability and validity of the Geriatric Depression Scale in depression in Parkinson's disease. J Neurol Neurosurg Psychiatry 2005;76:1445-7.

12. Casey AT, Crockard HA, Bland JM, Stevens J, Moskovich R, Ransford AO. Developmnet of a functional scoring system rheumatoid arthritis patients with cervical myelopathy. Ann Rheum Dis 1996 ;55:901-6.

13. Masthoff ED, Trompenaars FJ, Van Heck GL, Hodiamont PP, De Vries J. Validation of the WHO Quality of Life assessment instrument (WHOQOL-100) in a population of Dutch adult psychiatric outpatients. Eur Psychiatry 2005;20:465-73.

14. Huang IC, Wu AW, Frangakis C. Do the SF-36 and WHOQOL-BREF measure the same constructs? Evidence from the Taiwan population*. Qual Life Res 2006;15:15-24. 